\title{
Staged Approach for Petroclival Meningioma Resection
}

\author{
Georgios Klironomos ${ }^{1} \quad$ Amrit Chiluwal $^{1} \quad$ Amir Reza Dehdashti ${ }^{1}$ \\ ${ }^{1}$ Department of Neurosurgery, North Shore University Hospital at \\ Manhasset, Manhasset, New York, United States \\ Address for correspondence Georgios Klironomos, MD, MSc, PhD, \\ Department of Neurosurgery, North Shore University Hospital at \\ J Neurol Surg B 2019;80(suppl S3):S308-S309. \\ Manhasset, 300 Community Drive, Manhasset, NY 11030-3876, \\ United States (e-mail: geklironomos@gmail.com).
}

\begin{abstract}
Keywords

- petroclival meningioma

- staged approach

- anterior petrosectomy

- retrosigmoid craniotomy

Introduction Petroclival meningiomas represent very uncommon and challenging tumors. Surgical morbidity is high due to the difficult and complex approaches to this area. In the present video presentation, we demonstrate a staged-approach surgical resection for petroclival meningioma.

Patient and Methods A 47-year- old female was incidentally diagnosed with right sizable petroclival meningioma (-Fig. 1). The decision to proceed with a staged approach was made based on size and extension of the tumor to both the middle and posterior fossa. At the first stage we performed a right anterior petrosectomy with the patient in the supine position and the head turned 45 degrees to the left. Residual tumor was left behind along its inferior pole. At the second stage, 2 weeks after the 1st surgery, a right retrosigmoid craniotomy was performed with the patient placed on left park-bench position and the residual tumor was removed. The patient tolerated both stages very well without significant neurological deficits except a transient diplopia after the first stge. Postoperative magnetic resonance imaging (MRI) revealed gross total resection of the tumor.

Conclusion Staged approach for petroclival meningiomas represents a safe and effective surgical management, tolerable for the patient and more comfortable for the neurosurgeon.

The link to the video can be found at: https://youtu.be/QJJchjAwD5c.
\end{abstract}

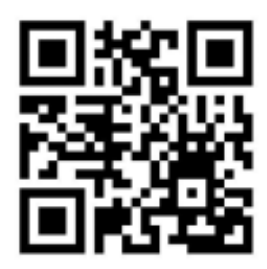

received

September 27, 2018 accepted

November 11, 2018

published online

February 28, 2019

www.thieme.com/skullbasevideos

www.thieme.com/jnlsbvideos

DOI https://doi.org/

10.1055/s-0039-1677849. ISSN 2193-6331. (c) 2019 Georg Thieme Verlag KG Stuttgart · New York
License terms

(c) (i) $\ominus$ (5) 


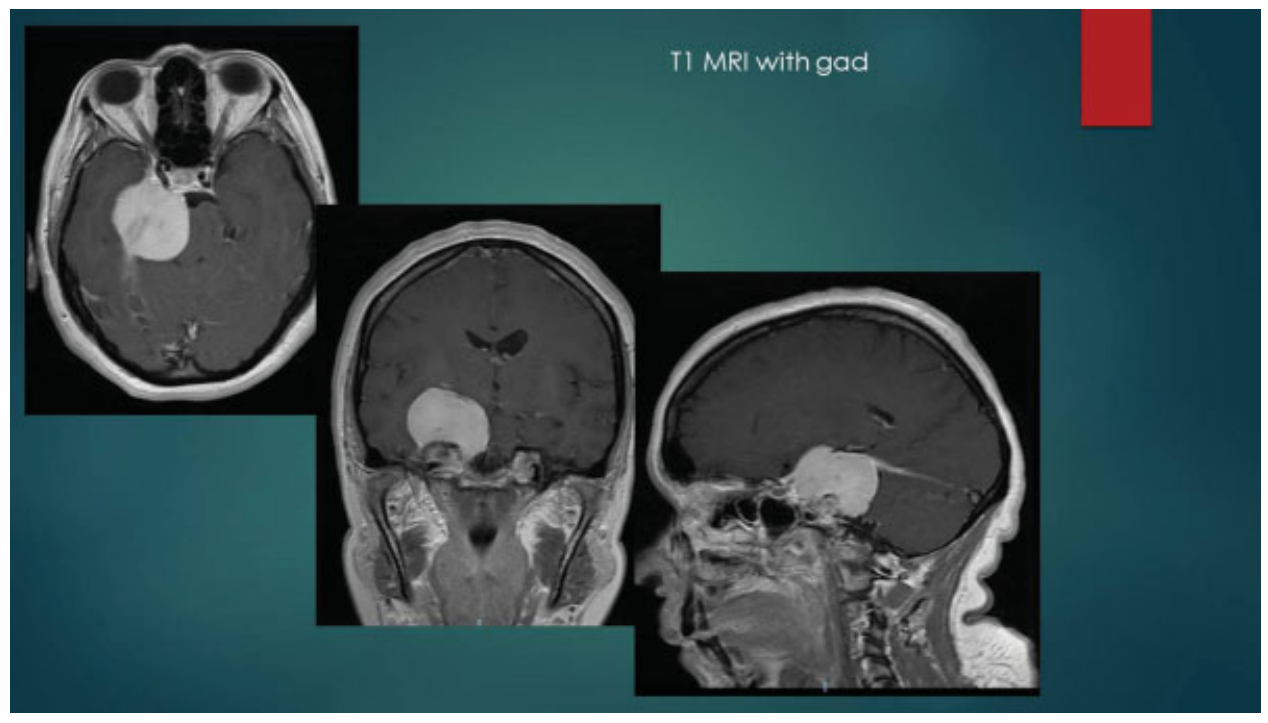

Fig. 1 MRI T1 + gad demonstrates a sizable petroclival meningioma. MRI, magnetic resonance imaging. 